\title{
Can activators enhance potentially developmental capacity of 2-cell blocked mouse embryos?
}

\author{
Mohammad Reza Darabi ${ }^{1}$, Parvindokht Bayat ${ }^{1}$, Ali Reza Shams ${ }^{2}$, Azindokht Nezhadi ${ }^{3}$, \\ Ali Faraji 4
}

Article History:
Received July 2013
Accepted September 2013
Available online September 2013
Keywords:
Activation
Arrest
Mouse
Two-Cell Embryo
Corresponding Author:
Mohammad Reza Darabi,
Department of Anatomy, Arak
University of medical sciences,
Arak, Iran
Tel: +988614173526
Email: m_darabi42@arakmu.ac.ir

\section{Article History:}

Received July 2013

Accepted September 2013

Available online September 2013

Keywords:

Arrest

Mouse

Email: m_darabi42@arakmu.ac.ir

\begin{abstract}
:
Objective: Two-cell block as a problem occurs in some couples referring to infertility center. This study was designed to compare the effect of different kinds of chemical activators on arrested mouse two-cell stage embryos in order to enhance cleavage and developmental formation rate.

Material and Methods: Following superovulation, the female mice were mated with males and positive vaginal plaque mice were euthanized 48 hours after hCG injection. Subsequently, 2cell embryos were collected and randomly cultured (in M16 medium) in six groups. Some embryos were washed and cultured as 1st group without any exposure. The remaining 2-cell stage embryos were exposed to $4^{\circ} \mathrm{C}$ for 24 hours in order to arrest in 2-cell stage for 2nd to 6th groups. The 2nd group was incubated immediately, while the 3rd group was exposed to $10 \mu \mathrm{M}$ Ionomycine for 3 minutes and the 4 th group was exposed to $10 \mathrm{mM}$ strontium for 5 minutes. The 5 th group was exposed to $\% 0.1$ Ethanol for 5 minutes and the 6th group to $\% 0.1$ Methanol for 3 minutes. Subsequently, all groups were incubated up to blastocyst stage.

Results: Data were analysed employing a one-way Anova test the results show that the rate of degenerated embryos is significantly different $(\mathrm{P}<0.05)$ between groups by low temperature $\left(4^{\circ} \mathrm{C}\right)$ exposure. The mean percentages of cleavage, blastocyst and hatched blastocyst formation rate in the 4th group were $80.9 \%, 69.2 \%$, and $46 \%$ respectively, showing a significant difference between groups.

Conclusion: This study shows that among different chemical activators used in this study, Strontium is the most powerful chemical activator to enhance cleavage and development of arrested two-cell embryos in the $4^{\text {th }}$ group.
\end{abstract}

1- Department of Anatomy, Arak University of Medical Sciences, Arak, Iran.

2- Department of Anatomy, Alborz University of Medical Sciences, Karaj, Iran.

3- Young Researchers and Elite Club, Arak Branch, Islamic Azad University, Arak, Iran.

4- Medicine Student, International Branch of Shahid Beheshti University of Medical Sciences \& Health, Tehran, Iran . 


\section{Introduction:}

Many different factors affect cleavage and development of in vitro produced embryos, some of which cause developmental arrest and infertility. Decreasing the growth and the developmental rate of embryo and arresting in certain step of development like pronuclear and two-cell stage (1) could be the reason of infertility in some couples referring to fertility and infertility center (1$2)$. few studies are performed in order to overcome two-cell blocks. For example, Matsukawa et al. showed that developments of two-cell block mouse embryos are attainable by polyaminocarboxylates with various metal ion stability constants, including EDTA and DTPA, whereas they are not attained by dipicolinic acid and deferoxamine, which are nonpolyaminocarboxylate metal ion chelators. EDTA caused maximum alleviation of the two-cell block (3).

Embryo can be stored at temperatures above freezing for only a limited period of time, although in comparison with freezing this technique is useful and more convenient (4). One of the features of in vitro-produced (IVP) mammalian embryos is the high frequency of early developmental failure thought to be brought on by sub-optimal culture environments $(2,5)$.

According to the typically low gene expression profiles of most genes and the level of specific maternal stores, early cleavage embryos, which are sensitive to their microenvironment, including suboptimal culture conditions may signal permanent embryo arrest at this stage of development as a means to prevent further development of low quality (abnormaldamaged) embryos (6). Reciprocal crosses between blocking and no blocking strains indicated that this in vitro 2 -cell block is a maternally regulated occurrence (7). In many animals, oocyte or follicular components assembled during oogenesis, control the earliest stages of development which occur independently from major activity of the embryonic genome (8).

During the past decades, intensive efforts have been made to elucidate the 2 -cell block. However, there is still little information on the mechanism of this event. It has often been assumed that block occurrence in early developing cultured embryos is due to the failure of zygotic gene activation (9). Twocell block is defined functionally as the inability of fertilized eggs to develop at in vitro post two-cell stage (10).

Different agents have been used to induce embryo activation, and the majority of the protocols used in nuclear transfer were adapted from previous studies describing oocyte parthenogenetic activation (11-12). Recently, Nasr-Esfahani et al. reported that artificial oocyte activation with Ionomycin can improve fertilization and cleavage rates in teratozoospermic patients (13).

Altering signal transduction pathways that regulate the rate of embryogenesis using Ethanol may influence preimplantation development. Ethanol at concentration more than $5 \%$ can elicit parthenogenetic activation of oocytes (14-16). In adition to ethanol, other alcholes including propandiol and methanol are potent parthenogenetic activators (17-19). Oocyte activation upon exposure to ethanol has been attributed to a transient rise in intracellular calcium due to an increase in membrane permeability to calcium (14-17). The possible role of $\mathrm{Ca} 2+$ in the stimulation of preimplantation development is accelerated by the increased rate of fluid accumulation during cavitation. Similar effect is reported for Calcium Ionophore, so that elevation of intracellular $\mathrm{Ca} 2+$ could also accelerate cavitation.

The molecular mechanisem underlying arrest in two-cell embryos is poorly understood. It was shown that the developmental arrest is maternally controlled (7, 20-21) and can be overcome by cytoplasm injection from nonblocking strains (22-23). It was shown that the loss of Cdc20 causes mouse embryogenesis to stop at the two-cell stage. In fact Cdc20 is essential for metaphase-to-anaphase transition.

Downs and et al. shows that D-glucose and hypoxantine alone had little inhibitory activity on embryo development. These two components interact in a synergistic manner to arrest embryos after first cleavage. The one-cell stage is unusually sensitive to the type and the concentration of energy source. The differences between blocking and non- 
blocking strain of mice may be related to different lactates which is apyrovate ratios necessary for balancing the reduction potential of the embryo(24).

In mammalian species, parthenogenetic development has been achieved by a variety of methods that induce $\mathrm{Ca} 2+$ transients (25). Although single $\mathrm{Ca} 2+$ elevations are induced by ethanol, calcium ionophore, or an electrical pulse are not sufficient to promote development. Multiple $\mathrm{Ca} 2+$ elevation, and post strontium $(\mathrm{Sr} 2+)$ treatment significantly enhance the development of oocytes (26-27) by mimicking sperminduced oscillations $(26,28)$. It is also possible that fluctuations in ion concentrations in the culture medium may cause the two-cell block in mice. The arrested two-cell stage embryos have significantly elevated the levels of both intracellular $\mathrm{pH}$ and intracellular free $\mathrm{Ca} 2+$ (29).

Methods used to maintain embryos in a metabolically inhibited but viable state, include cooling at refrigerated temperatures (short term storage), which is clearly simpler, more convenient and inexpensive than cryopreservation of embryos. Success with the storage of preimplantation embryos at temperatures from 0 to 7 has been reported for mice (30). There have been several studies about the effects of temporary storage at low temperatures on the development of fertilized mouse oocytes. DNA replication temporarily ceases in fertilized oocytes under storage at $4 \mathrm{oC}$ for $12 \mathrm{~h}$, but recovers immediately after return of oocytes to the normal temperature (37oC). Morphologically all oocyte treated at $4 \circ \mathrm{C}$ showed centering of the female and male pronuchlei or close association of female and male pronucli $6 \mathrm{~h}$ after return to $37 \mathrm{OC}(31$ $32)$. There are many efforts in order to minimize the effects of potentially extrinsic factors (like combination of medium and oxidative metabolite) on developmental arrest (3). Similarly intrinsic factors like genes, cytoplasmic factors, and chromosome reconstruction can terminate to arrest (33). Many protocols are used for oocyte activation and are divided into physical and chemical protocols. This study was designed to select the most powerful activator among the Ionomycine, Ethanol, Strontium and Methanol for embryo activation in order to enhance the growth and the development of two-cell arrested NMRI mouse embryo.

\section{Material \& Methods:}

All experiments were approved by the Arak University of Medical Sciences Animal Ethics. Experiments were conducted in accordance with the Iranian health and medical research council code of practice for the care and use of animals for scientific purposes. In this experimental study, 6 to 8 week-old mice (NMRI strain, $\mathrm{N}=200$ ) were recruited from Razi Vaccine \& Serum Research Institute and in order to get them adapt with the new environment, they were kept in the animal house of Arak University of Medical Sciences under standard conditions (12 h light, $12 \mathrm{~h}$ dark, $21 \pm 5{ }^{\circ} \mathrm{C}$ ) for one week (34-35). Female mice were injected intraperitoneally by 10 IU PMSG (Intervet Holland) for superovulation. After 48 hours, they were injected by 10 IU hCG) Organon Holland and coupled with male mice. Positive vaginal plug mice were separated and euthanized 48 hours after hCG injection by the cervical dislocation method (39). Two-cell stage embryos (Figure 1.a) were collected by flushing of oviduct, transferred to 25 microdroplets M16 medium (Sigma, M-7292) covered with mineral oil, after $3 \mathrm{X}$ washing. Subsequently, the collected embryos were divided into six groups: some embryos were washed and cultured as 1st group without any exposure. The remaining 2-cell stage embryos were exposed to $4^{\circ} \mathrm{C}$ (32) for 24 hours in order to arrest in 2-cell stage for 2 nd to 6 th groups (Fig. 1). The 2nd group was incubated immediately, while the 3rd group was exposed to $10 \mu \mathrm{M}$ Ionomycine (C-7522, Sigma) for 5 minutes and the 4th group was exposed to $10 \mathrm{mM}$ strontium for 5 minutes. The 5th group was exposed to \%7 Ethanol for 7 minutes and 6th group to \%0.1 Methanol for 3 minutes (36-41). The numbers of two-cell embryos in each group were 10-15 and for all, there was a minimum of 3 repeats with each group.

\section{Embryo evaluation:}

Evaluation was done at hours 48, 72 and 120 post culture by stereomicroscopy and the 
rate of degenerated, cleaved, morula, and blastocyst stage embryos were counted.

Statistical analysis:

All statistical analyses of this study were done by the statistical software SPSS version 11.5. The growth rate and the developmental parameters of embryos were analyzed by a one-way ANOVA test. Significant differences between groups were determined by Post Hoc analyses.

\section{Results:}

Effect of $4^{\circ}$ C on development of mouse twocell Embryos:

Data analysis by a one-way ANOVA test shows that embryos (Table 1) exposed to low temperatures (group 2), develop to the blastocyst stage nearly 12 - 24 hours later than the embryos that were not exposed in group 1 (data was not shown). The results of the growth and the development of the embryos are shown in tables 1 and 2 . The rate of the degenerated embryo in group 2 (exposed to low temperatures) is significantly different from the embryos in other groups except group $6(\mathrm{P}=0.999)$. The rate of embryos arrested in 3-4-cell stage in group 2 is more than other groups but these differences are not significant. The rate of the embryos arrested in 5-8-cell stage (Figure 1.b) in group 2 is more than other groups, but these differences are not significant. The rate of morola (Fig.1.c) in group 2 is lower than other groups (Table 1 ) and the differences are significant except for group $6(\mathrm{P}=0.395)$. The rate of the blastocyst stage embryos (Figure 1.d) in group 2 is significantly lower than other groups except groups 2 \& $6(\mathrm{P}=0.930)$ (Table 2). The rate of hatched blastocyst (Figure 1.d) is significantly lower in group 2 compared with group 1 except with group 5 which is not significant $(\mathrm{P}=0.322)$ (Table 2$)$. In a similar manner the cleavage rate in group 2 is significantly lower than the other groups except group $6(\mathrm{P}=0.999)$.

Effect of Ionomycine on arrested two-cell Embryos:

Data analysis by a one-way ANOVA test shows that the rate of the degenerated embryos (Table 1) in group 3 (exposed to Ionomycine) is significantly different from the groups $1 \& 2 \& 6$, but the differences are not significant with groups $4 \& 5$. The rate of morale stage embryos in group 3 is not significantly lower than groups $4 \& 5$, but is significantly more than groups $2 \& 6$. The rate of blastocyst stage embryos in group 3 (Table 2) is significantly more than groups 2 $\& 6$, but lower than groups $1 \& 4 \& 5$. The rate of hatched blastocyst in group 3 has not significant differences with groups $2 \& 5$, but its difference from groups $1 \& 4 \& 6$ is significant. The cleavage rate in group 3 is not significantly lower than groups $4 \& 5$, but is significantly more than groups $2 \& 6$.

Effect of strontium on arrested two-cell embryos:

This study shows that the rate of the degenerated embryos (Table 1) in group 4 (exposed to Strontium) has significant differences from groups $2 \& 6$, but the differences are not significant from groups $1 \& 3 \& 5$ ( $>>0.096)$. The rate of morula stage embryos in group 4 is not significantly more than groups $3 \& 5$, but is significantly more than groups $2 \& 6$. The rate of blastocyst stage embryos in group 4 is significantly more than groups $2 \& 3 \& 6$, but its difference from group 5 is not significant $(\mathrm{P}=0.716)$. The rate of hatched blastocyst in group 4 (Table 2) is significantly more than groups 2 \& $3 \& 6$, but its difference from group 5 is not significant $(\mathrm{P}=0.162)$. The cleavage rate in group 4 (Table 2 ) is not significantly more than groups $3 \& 5$, but significantly more than groups $2 \& 6$.

Effect of Ethanol on arrested two-cell Embryos:

Our data shows that the rate of the degenerated embryos (Table 1) in group 5 (exposed to Ethanol) is significantly lower than groups $2 \& 6$, but is not significantly different from groups $3 \& 4$ ( $>>0.359)$. The rate of morula stage embryos in group 5 is significantly more than groups $2 \& 6$, but is significantly different from groups $3 \& 4$. The rate of blastocyst stage embryos in group 5 (Table 2) is significantly more than groups 2 \& $3 \& 6$, but its difference with group 4 is not significant $(\mathrm{P}=0.716)$. The rate of hatched blastocyst in group 5 is not significantly different from groups $2 \& 3 \& 4$ ( $>>0.162)$ and significantly more than group 6 . The cleavage rate in group 5 (Table 2) is significantly more than groups $2 \& 6$, but is 
not significantly different from groups $3 \& 4$ ( $\mathrm{P}>0.359)$.

Effect of Methanol on arrested two-cell Embryos:

Data analysis shows that the rate of the degenerated embryos (Table 1) in group 6 (exposed to Methanol) is significantly more than other groups except group 2 which has no significant differences from group 6 $(\mathrm{P}=0.999)$. The rate of morula stage embryos in group 6 is significantly lower than other groups except group 2 which has no significant differences from group 6 $(\mathrm{P}=0.359)$. The rate of blastocyst stage embryos in group 6 (Table 2) is significantly lower than other groups except group 2 which has no significant differences from group $6(\mathrm{P}=0.930)$. The rate of hatched blastocyst in group 6 is significantly lower than other groups $(\mathrm{P}=0.004)$. The cleavage rate in group 6 is significantly lower than other groups except group 2 which has no significant differences from group 6 $(\mathrm{P}=0.999)$. Each treatment repeated 3 to 5 times.

\section{Discussion:}

Although the effects of chemical activators on cleavage and the development of parthenogenic oocyte and early embryos have been investigated previously, but to the best of our knowledge, the effect of these materials on cleavage and the development of arrested two-cell embryos had not been investigated. A previous study shows that mammalian egg activation can also be achieved by a variety of artificial stimuli (42). Exposing embryos to the low temperature of $4{ }^{\circ} \mathrm{C}$, decreased cellular metabolism and protein synthesis of embryos and thus, the metabolic transport system was affected. Probably in this condition, embryotrophic factors change to embryotoxic factors after a short time and increase the sensitivity of embryos to unfavorable compounds of culture system (40). Similarly in this study, the rate of degenerated embryos is increased by exposure to a low temperature in group 2 (table 1). Although Calcium Ionophore, Strontium, Ethanol and Methanol could decrease the rate of degenerated embryos by increasing the rate of cleavage and development. (this sentence is not complete!! Although introduces a dependent clause which should be completed with an independent clause)

The arrested and degenerated embryos exposed to $4^{\circ} \mathrm{C}$ were increased, and they reached the blastocyst stage later than the control group even though they would survive if they were incubated again in optimal conditions and were exposed to effective activators. Manipulating $\mathrm{Ca} 2+$ signaling patterns during egg activation can severely alter the pre-implantation development and the implantation outcome (43). $\mathrm{Ca} 2+$ transients can affect the morphological, biochemical and the developmental events of egg activation. Similarly $\mathrm{Ca} 2+$ signaling at fertilization is a vital event for switching the oocytes from meiosis to mitosis (2). Probably because of the low effectiveness in activation and toxic effect of Methanol, the degenerated embryos did not show any significant differences between groups $2 \& 6$.

With effects of Calcium Ionophore on arrested two-cell embryos (3rd group), more embryos developed to blastocyst related to 2 nd group (Table 2), so the rate of degenerated embryos decreased in the 2 nd $\&$ the 6th group and had no significant differences from the 4 th $\&$ the 5 th group.

Zhang et al. showed that strontium is the most efficient parthenogenic agent for activating mouse ovulated and nuclear transplanted eggs. Their results are in line with findings of the present study (44). According to the above-mentioned study, the mechanism by which strontium causes $\mathrm{Ca} 2+$ oscilations in early embryos remains unknown.

As an important step in the activation procedure, Yamazaki evaluated the effect of strontium for oocyte activation on in vitro and in vivo developmental capacity of bovine reconstructed embryos. Their results showed that strontium can be used as an activation agent in bovine cloning procedures and that activation with a combination of Strontium and Ionomycin increased the in vitro developmental capacity of reconstructed embryos. In their study the cleavage rate with $20 \mathrm{mM}$ of strontium was $42.9 \%$, so $12.6 \%$ of them reached the blastocyst stage (45). 
Krivokharchenko et al. used $2 \mathrm{mM}$ strontium for 2 hours, in order to activate rat oocytes parthenogenetically at the preimplantation stage. The mean percent of cleavage rate was $93 \%$ which is higher than the results in the present study (81\%). This can be attributed to the parthenogenesis, arrest and different dosages of Strontium used in their study (38). Their lower cleavage rate is probably as a result of the effect of manipulations and stresses during nuclear transfer and the differences between two species.

Rogers et al. used 7\% ethanol for 7 minutes in order to activate parthenogenetic oocytes. The rates of cleavage, morula and blastocyst formation rate were 83,79 and $72 \%$ respectively which were significantly more than the results of the present study (respectively 78, 59 and 35\%) (39), possibly because of the low temperature exposure in the present study.

It was shown that the short-term (15 $\mathrm{min})$ and the long-term $(2 \mathrm{~h})$ strontium treatments were superior to stimulation by ethanol for parthenogenetic activation. The mean percentage of cleavage 12-14 $\mathrm{h}$ after hCG injection was 93\% (38) while in our study it was $79 \%$. This difference may be due to the different strain of rats and mice and also the low temperature exposure in this study.

Yamazaki et al. showed that strontium can be used as an activation agent in bovine cloning procedures meanwhile activation with combination of strontium and ionomycin increased the in vitro developmental capacity of reconstructed embryos. In their study, the cleavage rate with $20 \mathrm{mM}$ of strontium treatment was 42.9 $\%$, so $12.6 \%$ of them reached the blastocyst stage (45). Our different results are probably because of the different dosage of strontium used in this study.

\section{Conclusion:}

In conclusion, the present study showed that Strontium and Ethanol are more effective than the other chemical activators used in this study, so for cleavage, development to blastocyst and hatched blastocyst by Strontium is more than Ethanol but their differences are significant only for hatched blastocyst.
Finally, we recommend that the effects of other activators and their combination on two-cell arrested embryonic development and following probably chromosomal abnormalities be investigated in the future.

\section{Acknowledgments:}

The authors are thankful to the Department of Anatomy of Arak University of Medical Science for their technical help. Thanks also go to Mr. Bahram Nezhadi for his financial support. The results described in this paper were part of student thesis.

\section{Conflict of interest statement :}

There is no conflict of interest between the authors and none of them has any financial or personal relationships that could have inappropriately influenced or biased the contents of this paper.

Figure1. Shows mouse embryos at different stages of development $(\times 400$ magnification)

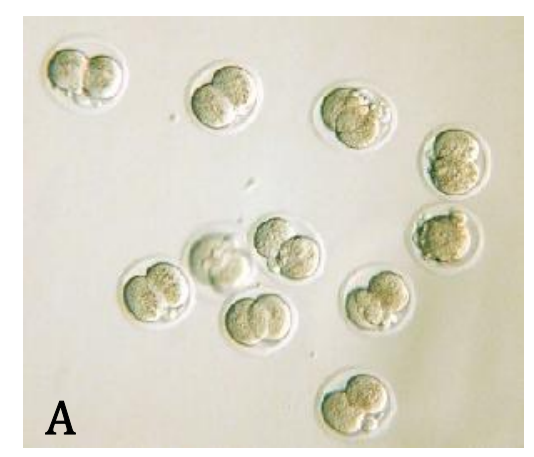

Arrested two-cell embryo: (A)

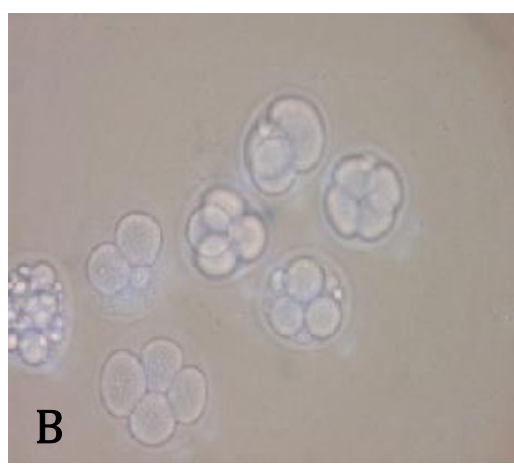

3-8 cell stage embryos (B) 


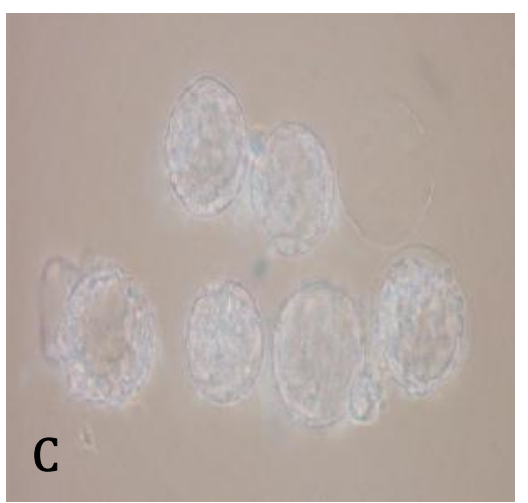

morula stage embryos (C)

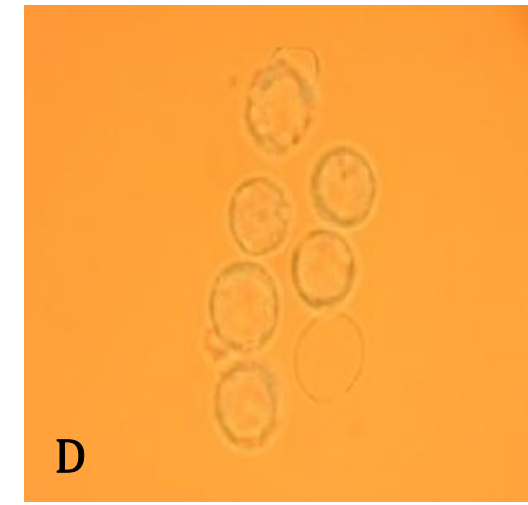

blastocyst and hatched blastocyst stage embryos (D).

Table 1. The cleavage of arrested mouse two-cell embryos by Ionomycine (Group 3), Strontium (Group 4), Ethanol (Group 5) and Methanol (Group 6) exposure.

\begin{tabular}{cccccc}
\hline & Degenerated $(\mathrm{n})$ & $3-4$ Cell $(\mathrm{n})$ & $5-8$ Cell(n) & Morula(n) & Total \\
\hline Gr.1 & $11.9 \pm 3.6(15)$ & $6.4 \pm 2.2(8)$ & $7.5 \pm 4.3(9)$ & $74.2 \pm 3.7(94)$ & 126 \\
\hline Gr.2 & $44.6 \pm 7.5(87)$ & $11.2 \pm 3.8(23)$ & $13.3 \pm 5.5(27)$ & $30.8 \pm 7(59)$ & 196 \\
\hline Gr.3 & $28.4 \pm 3.3(32)$ & $11.5 \pm 2.2(13)$ & $5.6 \pm 2.7(6)$ & $54.4 \pm 4.4(62)$ & 113 \\
\hline Gr.4 & $21 \pm 3.9(22)$ & $7.7 \pm 3.9(8)$ & $11.2 \pm 6.5(11)$ & $63(60 \pm 5.9)$ & 104 \\
\hline Gr.5 & $22 \pm 3.6(37)$ & $10.7 \pm 2.8(18)$ & $8.5 \pm 2.3(15)$ & $59 \pm 4.3(100)$ & 170 \\
\hline Gr.6 & $45.7 \pm 6.1(93)$ & $15.4 \pm 6.4(30)$ & $13.1 \pm 4.8(26)$ & $25.8 \pm 2.3(52)$ & 201 \\
\hline
\end{tabular}

Value in the parenthesis shows the number of embryos.

Table 2. Development of arrested mouse two-cell embryos by Ionomycine (Group 3), Strontium (Group 4), Ethanol (Group 5) and Methanol (Group 6) exposure.

\begin{tabular}{|c|c|c|c|c|}
\hline & Blastocyst (n) & Hatched(n) & Cleavage(n) & Total \\
\hline Gr.1 & $61.4 \pm 6(78)$ & $44 \pm 8.4(55)$ & $88.1 \pm 3.6$ & 126 \\
\hline Gr.2 & $13.7 \pm 3.2(26)$ & $12.2 \pm 2.5(24)$ & $55.4 \pm 7.5$ & 196 \\
\hline Gr.3 & $28.7 \pm 2.2(32)$ & $12.7 \pm 2(14)$ & $71.6 \pm 3.3$ & 113 \\
\hline Gr.4 & $40(38.6 \pm 2.3)$ & $23(22.2 \pm 1.6)$ & $79 \pm 3.9$ & 104 \\
\hline Gr.5 & $35.8 \pm 2.8(61)$ & $16.5 \pm 3.7(29)$ & $78 \pm 3.6$ & 170 \\
\hline Gr.6 & $12.1 \pm 2(24)$ & $4.1 \pm 1.6(8)$ & $54.3 \pm 6.1$ & 201 \\
\hline
\end{tabular}

Value in the parenthesis shows the number of embryos. 


\section{References:}

1. Heindryckx, B et al. Treatment option for sperm- or oocyte-related fertilization failure: assisted oocyte activation following diagnostic heterologous ICSI. Hum Reprod, 2005; 20(8): 2237-41.

2. Betts, D.H. and W.A. King. Telomerase activity and telomere detection during early bovine development. Dev Genet.1999; 25(4): 397-403.

3. Matsukawa, T et al. Alleviation of the two-cell block of ICR mouse embryos by polyaminocarboxylate metal chelators. Reproduction. 2002; 124(1):65-71.

4.Kasai, M., K. Niwa, and A. Iritani, Effects of various cryoprotective agents on the survival of unfrozen and frozen mouse embryos. J Reprod Fertil. 1981; 63(1):175-80.

5. Johnson, M.H. and M.H. Nasr-Esfahani, Radical solutions and cultural problems: could free oxygen radicals be responsible for the impaired development of preimplantation mammalian embryos in vitro? Bioessays. 1994; 16(1): 31-8.

6. Betts, D.H. and W.A. King. Genetic regulation of embryo death and senescence. Theriogenology. 2001; 55(1):171-91.

7. Goddard, M.J. and H.P. Pratt, Control of events during early cleavage of the mouse embryo: an analysis of the '2-cell block'. J Embryol Exp Morphol. 1983; 73: 111-33.

8. Qiu, J.J., et al., Delay of ZGA initiation occurred in 2-cell blocked mouse embryos. Cell Res. 2003; 13(3):79-85.

9. Johnson, M.H. The molecular and cellular basis of preimplantation mouse development. Biol Rev Camb Philos Soc. 1981; 56(3): 463-98.

10. Biggers, J.D. Reflections on the culture of the preimplantation embryo. Int J Dev Biol. 1998; 42(7): 879-84.

11. Fissore, R.A et al. Initiation and organization of events during the first cell cycle in mammals: applications in cloning. Cloning. 1999; 1(2): 89-100.

12. Revazova, E.S et al. Patient-specific stem cell lines derived from human parthenogenetic blastocysts. Cloning Stem Cells. 2007; 9(3): 432-49.

13. Nasr-Esfahani, M.H et al. Artificial oocyte activation in severe teratozoospermia undergoing intracytoplasmic sperm injection. Fertil Steril. 2008; 90(6): 2231-7.

14. Cuthbertson, K.S. and P.H. Cobbold. Phorbol ester and sperm activate mouse oocytes by inducing sustained oscillations in cell Ca2+. Nature. 1985; 316(6028): 541-2.

15. Surani, M.A., S.C. Barton, and M.L. Norris. Development of reconstituted mouse eggs suggests imprinting of the genome during gametogenesis. Nature. 1984; 308(5959): 548-50. 
16. Colonna, $\mathrm{R}$ et al. Effects of protein kinase $\mathrm{C}$ stimulation and free $\mathrm{Ca} 2+$ rise in mammalian egg activation. Gamete Res. 1989; 24(2): 171-83.

17. Cuthbertson, K.S., D.G. Whittingham, and P.H. Cobbold. Free Ca2+ increases in exponential phases during mouse oocyte activation. Nature. 1981; 294(5843): 754-7.

18. Cuthbertson, K.S. Parthenogenetic activation of mouse oocytes in vitro with ethanol and benzyl alcohol. J Exp Zool.1983; 226(2): 311-4.

19. Shaw, J.M. and A.O. Trounson, Parthenogenetic activation of unfertilized mouse oocytes by exposure to 1,2-propanediol is influenced by temperature, oocyte age, and cumulus removal. Gamete Res. 1989; 24(3): 269-79.

20. Loutradis, D., D. John, and A.A. Kiessling. Hypoxanthine causes a 2-cell block in randombred mouse embryos. Biol Reprod. 1987 ;37(2): 311-6.

21. McLaren, A., The developmental history of female germ cells in mammals. Oxf Rev Reprod Biol. 1988; 10: 162-79.

22. Muggleton-Harris, A., D.G. Whittingham, and L. Wilson. Cytoplasmic control of preimplantation development in vitro in the mouse. Nature. 1982; 299(5882): 460-2.

23. Pratt, H.P. and A.L. Muggleton-Harris. Cycling cytoplasmic factors that promote mitosis in the cultured 2-cell mouse embryo. Development. 1988; 104(1): 115-20.

24. Downs, S.M. and M.P. Dow. Hypoxanthine-maintained two-cell block in mouse embryos: dependence on glucose and effect of hypoxanthine phosphoribosyltransferase inhibitors. Biol Reprod. 1991; 44(6): 1025-39.

25. Schultz, R.M. and G.S. Kopf. Molecular basis of mammalian egg activation. Curr Top Dev Biol. 1995; 30: 21-62.

26. Kline, D. and J.T. Kline, Repetitive calcium transients and the role of calcium in exocytosis and cell cycle activation in the mouse egg. Dev Biol. 1992; 149(1): 80-9.

27. Bos-Mikich, A., K. Swann, and D.G. Whittingham, Calcium oscillations and protein synthesis inhibition synergistically activate mouse oocytes. Mol Reprod Dev. 1995; 41(1): 8490 .

28. Fissore, R.A., M.M. Reis, and G.D. Palermo, Isolation of the Ca2+ releasing component(s) of mammalian sperm extracts: the search continues. Mol Hum Reprod. 1999; 5(3): 189-92.

29. Lane, M., T.E. Ludwig, and B.D. Bavister, Phosphate induced developmental arrest of hamster two-cell embryos is associated with disrupted ionic homeostasis. Mol Reprod Dev. 1999; 54(4): 410-7.

30.Tsuchiya, $\mathrm{H}$ et al. Short-term preservation of mouse oocytes at 5 degrees C. Exp Anim. 2001; 50(5): 441-3. 
31. Sakurai, T., M. Kimura, and M. Sato. Temporary developmental arrest after storage of fertilized mouse oocytes at 4 degrees $\mathrm{C}$ : effects on embryonic development, maternal mRNA processing and cell cycle. Mol Hum Reprod. 2005; 11(5): 325-33.

32. Kwon, I.K., K.E. Park, and K. Niwa. Activation, pronuclear formation, and development in vitro of pig oocytes following intracytoplasmic injection of freeze-dried spermatozoa. Biol Reprod. 2004; 71(5): 430-6.

33. Sharan, S.K et al. BRCA2 deficiency in mice leads to meiotic impairment and infertility. Development. 2004.;131(1):131-42.

34. Fukuhara, R et al. Erythrocytes counteract the negative effects of female ageing on mouse preimplantation embryo development and blastocyst formation. Hum Reprod. 2008; 23(9): 2080-5.

35. Biggers, J.D. and M.C. Summers, Impact of hyperglycemia on early embryo development and embryopathy: in vitro experiments using a mouse model. Hum Reprod. 2008; 23(12): 2874-5.

36. Revazova, E.S., et al., HLA homozygous stem cell lines derived from human parthenogenetic blastocysts. Cloning Stem Cells. 2008; 10(1): 11-24.

37. Ma, S.F et al. Parthenogenetic activation of mouse oocytes by strontium chloride: a search for the best conditions. Theriogenology. 2005; 64(5): 1142-57.

38. Krivokharchenko, A et al. Development of parthenogenetic rat embryos. Biol Reprod, 2003; 68(3): 829-36.

39. Rogers, N.T et al. The absence of a $\mathrm{Ca}(2+)$ signal during mouse egg activation can affect parthenogenetic preimplantation development, gene expression patterns, and blastocyst quality. Reproduction. 2006; 132(1): 45-57.

40. Grabiec, A., A. Max, and M. Tischner, Parthenogenetic activation of domestic cat oocytes using ethanol, calcium ionophore, cycloheximide and a magnetic field. Theriogenology. 2007; 67(4): 795-800.

41. Nandedkar, P et al. Parthenogenesis and somatic cell nuclear transfer in sheep oocytes using Polscope. Indian J Exp Biol. 2009; 47(7): 550-8.

42. Swann, K. and J.P. Ozil. Dynamics of the calcium signal that triggers mammalian egg activation. Int Rev Cytol.1994; 152: 183-222.

43. Ozil, J.P. and D. Huneau, Activation of rabbit oocytes: the impact of the $\mathrm{Ca} 2+$ signal regime on development. Development. 2001; 128(6): 917-28.

44. Zhang, D et al. Strontium promotes calcium oscillations in mouse meiotic oocytes and early embryos through InsP3 receptors, and requires activation of phospholipase and the synergistic action of InsP3. Hum Reprod. 2005; 20(11): 3053-61. 
45. Yamazaki, W., et al., Use of strontium in the activation of bovine oocytes reconstructed by somatic cell nuclear transfer. Zygote. 2005; 13(4): 295-302. 